\title{
ESTRATEGIAS ÓPTIMAS PARA LA REDUCCIÓN DE PERDIDAS DE AGUA EN SISTEMAS DE ABASTECIMIENTO
}

\author{
Antonio Vela, Fernando Martínez, Jorge García-Serra, Rafael Pérez \\ Unidad Docente Mecánica de Fluidos \\ Departamento de Ingeniería Hidráulica y Medio Ambiente \\ Universidad Politécnica de Valencia
}

\begin{abstract}
RESUMEN: La justificada y creciente preocupación por mejorar el aprovechamiento del agua en los sistemas de abastecimiento exige aumentar la calidad y rendimiento de dichos sistemas. El presente artículo presenta una serie de técnicas encaminadas a reducir las pérdidas de agua e incrementar la eficiencia de los abastecimientos. La metodología propuesta propone criterios de selección y optimización de las estrategias que deben ser aplicadas para conseguir estos objetivos de forma óptima. En este sentido se establece una jerarquía en base a criterios técnicos y de rentabilidad económica, que con la ayuda de modelos matemáticos y la debida coordinación con las demás funciones de la gestión y explotación del abastecimiento, permitirá confeccionar un programa integral para alcanzar y mantener un estado óptimo de funcionamiento del sistema. Asimismo, se recomienda efectuar un análisis completo del sistema hidráulico que, a través de su diagnóstico, determine las causas que disminuyen su seguridad y rendimiento, de modo que puedan optimizarse económicamente las medidas necesarias para cumplir las restricciones de riesgo máximo aceptable y eficiencia mínima exigida en el aprovechamiento de los recursos hídricos y energéticos, cada vez más escasos.
\end{abstract}

\section{INTRODUCCIÓN}

Los sistemas de abastecimiento de agua, pueden definirse como los encargados de garantizar el transporte y distribución de agua desde los puntos de captaciónproducción hasta las diferentes acometidas de los abonados y otros puntos de consumo, con una calidad de suministro mínima aceptable.

Obviamente la definición anterior precisa de una concreción mayor, tanto de los términos "garantía de transporte y distribución" y "calidad de suministro", como de los requisitos mínimos que deben exigirse a dichos sistemas en su concepción primero y posterior funcionamiento.

Entre los objetivos fundamentales del control y explotación de todo sistema hidráulico en general, y de los abastecimiento de agua en particular, destacan singularmente tres, que serían :

1. Control de calidad del agua aportada y de las condiciones de suministro.

2. Control de las pérdidas de agua.

3. Control de los costes de mantenimiento y explotación del servicio.

La preocupación creciente por la mejora continua de la eficacia y eficiencia de los sistemas encargados de cumplir satisfactoriamente dichos objetivos, exige cada vez más la introducción de metodologías y tecnologías apropiadas que ayuden al personal responsable a lograrlo. En particular, uno de los grandes problemas es el bajo rendimiento de los sistemas de distribución de agua, en torno al $60 \%$, (Estadísticas de la AEAS, 19871990) y su reducida fiabilidad en comparación con otras instalaciones. Las medidas que se proponen a continuación tienen la finalidad de ayudar a garantizar el éxito en lo que podría denominarse "la batalla contra las pérdidas de agua", que debe constituir actualmente un verdadero reto de todo el sector a nivel nacional e internacional.

Generalmente el estudio de los sistemas de abastecimiento ha contemplado solamente los aspectos relativos al diseño, cálculo, análisis y operación de los sistemas hidráulicos y en cambio es sabido que no es suficiente, en general, con un buen diseño del sistema (incluso óptimo) para las necesidades previstas en esa primera y fundamental etapa, sino que además debe comprobarse efectiva y continuadamente que el sistema se encuentra dentro de las especificaciones técnicas consideradas en su proyecto, y que además cumple los requisitos exigidos por la normativa vigente, en materia de calidad de agua sobre todo, pero también en cuanto al caudal y presión demandados por los usuarios.

En este sentido, la respuesta del sistema puede no ser la adecuada, bien transitoriamente o incluso continua- 
damente en algunos puntos de consumo. Las causas de estas deficiencias pueden ser varias y no sólo responsabilidad del diseño inicial.

Por otro lado, y al margen de la comprobación y análisis de la encacia del sistema para desempeñar las funciones encomendadas al mismo con las consideraciones y requisitos establecidos, debe también determinarse con rigor la eficiencia con que dicho sistema alcanza los objetivos marcados.

Esta segunda característica no es menos importante, si se tiene en cuenta que condiciona la primera, es decir, condiciona la eficacia del sistema hidráulico, limitando su capacidad de respuesta y su adaptabilidad ante las diferentes solicitaciones que se presenten a lo largo del tiempo, con el inevitable efecto del deterioro progresivo de sus componentes. Pero además una menor eficiencia del sistema hidráulico requiere un volumen de recurso hídrico mayor del necesario, lo que siendo un recurso bastante escaso es ya preocupante, y conlleva también unos consumos mayores de energía, costes de explotación adicionales e inversiones cuantiosas para compensar parcial o totalmente las deficiencias ocasionadas por unos bajos rendimientos volumétrico y energético.

\section{DIAGNOSTICO DE UN SISTEMA HIDRÁULICO A PRESIÓN}

\section{Clasificación de los consumos en una red}

Para resolver eficazmente los problemas planteados y realizar una gestión y una explotación óptimas, es estrictamente necesario $y$ fundamental cuantificar $y$ determinar las causas que han generado esa problemática, lo que se traduce en la realización de un diagnóstico completo y detallado.

El primer paso supone llevar a cabo el balance hídrico del abastecimiento en cuestión. Deben conocerse con cierta precisión las entradas de agua al sistema, así como su destino definitivo, es decir, la clasificación de los consumos de agua según su uso y fines concretos. Todos los estudios de diseño y análisis de un sistema deben partir de la información básica anterior, y por supuesto del diagnóstico técnico del sistema.

El caudal inyectado debe ser medido y registrado continuamente, pues es fundamental en el diseño y diagnóstico de las instalaciones de aprovisionamiento y almacenamiento. La previsión de dicho valor permite planificar y explotar adecuadamente el conjunto del abastecimiento en un futuro próximo y también a largo plazo.

Definidos convenientemente los aportes a la red, corresponde ahora determinar los puntos de consumo, los volúmenes extraídos y su destino. El volumen de agua suministrado por la red puede clasificarse en dos grandes grupos, como muestra la Figura 1, registrado y no registrado. El primero ha sido medido mediante aparatos insertados en las conducciones de acometida a los diferentes consumidores.

La suma de ambos, por continuidad, resulta ser el total de agua suministrada. Así pues el volumen no registrado se calcula mediante simple diferencia entre el volumen total inyectado y el volumen registrado. El balance en volúmenes de agua, referidos a un mismo período de tiempo, será el siguiente:

$V_{\text {TOTAL }}=V_{\text {RLGISTRADO }}+V_{\text {NO REGISTRADO }}$

El $V_{\text {TOtal }}$ y el $V_{\text {ReGistrado }}$ se obtienen a partir de las mediciones realizadas, son pues dato, y permiten por diferencia obtener el volumen restante:

$V_{\text {NO REGISTRADO }}=V_{\text {TOTAL }}-V_{\text {REGISTRADO }}$

Análogamente a como se ha procedido con los volúmenes, podría efectuarse un balance similar en caudales. Sin embargo, así como la medición del caudal aportado en un determinado instante no entraña hoy dificultad alguna mediante la inserción de caudalímetros en las conducciones principales de inyección, no ocurre lo mismo con los caudales demandados por los numerosos usuarios. La razón fundamental del registro del volumen y no del caudal reside en que la facturación del consumo se hace en base al volumen registrado.

El volumen registrado total es la adición de los volúmenes registrados en los diferentes puntos de consumo, de los cuales se conoce su ubicación espacial y también se tiene información acerca del tipo de uso. Una clasificación interesante es la mostrada en la citada Figura 1, donde se distinguen los usos domésticos, industrial, comercial, institucional y público.

La clasificación anterior obedece a dos criterios básicos:
- $\quad$ La susceptibilidad de un consumo de poder ser registrado.
- $\quad$ La causa que origina el consumo.

El balance de la Figura 1 debería aplicarse a los subsistemas, zonas o sectores que constituyen el sistema global. Mediante la realización de estos balances sectoriales, pueden emitirse diagnósticos más precisos y, conocidos los rendimientos de cada subsistema, establecer una estrategia adecuada para su mejora planificada en el tiempo. Este es el método más efectivo y eficiente, pues permite aplicar las medidas más convenientes a cada sector y realizar con rentabilidad máxima las inversiones en los sectores más necesitados. 


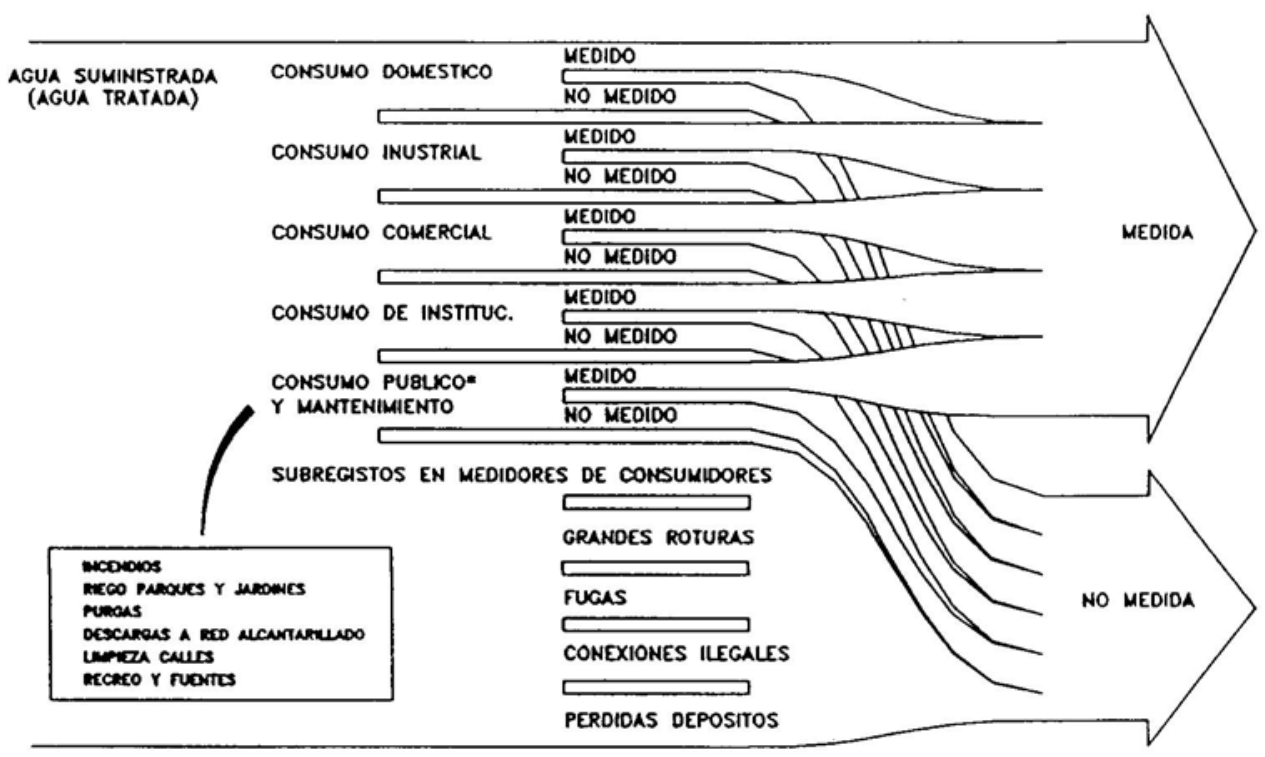

Figure 1. Classificación de los consumos en un abastecimiento de agua

Aplicar un programa de mejora del rendimiento y estado del abastecimiento a todo el sistema, sin ninguna estrategia espacial, aparte de tener mucha menos eficacia, supondría una inversión y un coste enormes y también una rentabilidad dudosa, en cualquier caso menor que la de unas inversiones seleccionadas y aplicadas sólo en ciertos sectores y elementos.

Las Figuras 1 y 2, ilustran estas clasificaciones bastante bien, siendo su trazado meramente orientativo $\mathrm{y}$ cualitativo, que no cuantitativo.

El volumen no registrado se debe analizar en base a las causas que impiden su registro en los contadores, lo que ya permite preseleccionar las posibles soluciones:

\section{a) Volumen no controlado debido a la ausencia de contador en el punto de consumo}

En este caso, la solución es evidente: deberá instalarse un aparato de medida en dicha acometida, pues aunque dicho volumen nunca vaya a ser facturado, sí debe ser controlado, por dos razones:

\section{- $\quad$ Aumentar el volumen de agua cuyo destino es conocido y controlado \\ - $\quad$ Evitar el despilfarro}

\section{b) Volumen no registrado por avería y error en contadores}

Una causa fundamental de que una parte del consumo que ha atravesado un aparato de medida no sea contabilizado, es el subregistro por error en el mismo. Aunque este volumen no constituye en sí mismo ninguna pérdida, debe reducirse al máximo, tanto para repercutir equitativamente el coste del servicio de agua entre los abonados al mismo, como para poder distinguir con rigor volúmenes no registrados, pero útiles, de las verdaderas pérdidas. Su cuantía puede rebasar el $15 \%$ del volumen registrado.

Para detectar lo más rápidamente posible el fallo o error de un contador deberían implantarse sistemas de telemedida de los mismos, lo que además de ahorrar considerables costes de lectura y facturación de consumos, permitiría reducir considerablemente el volumen infrarregistrado o simplemente no registrado por avería del contador.

\section{c) Volumen consumido en usos públicos}

Este grupo constituye el volumen utilizado en limpieza de calles, riego de jardines públicos, fuentes públicas, descargas en la red de alcantarillado, extinción de incendios, purgas y similares, etc., denominado consumo público y tampoco suele registrarse. En este caso debería controlarse su cuantía y en muchos casos puede estimarse su valor, bien por medidas periódicas del caudal o por contabilización del número de horas de uso. $\mathrm{Su}$ magnitud se estima en torno al $10 \%$ del volumen total.

\section{d) Volumen no registrado restante}

Este volumen constituye una verdadera pérdida, como se desprende del análisis efectuado. Es muy difícil de cuantificar, a no ser indirectamente por diferencia entre el volumen aportado y el resto de volúmenes citados; de ahí la importancia de medir el resto de volúmenes. Además, interesa conocer su composición, su variación con el tiempo y su relación con la presión existente. 


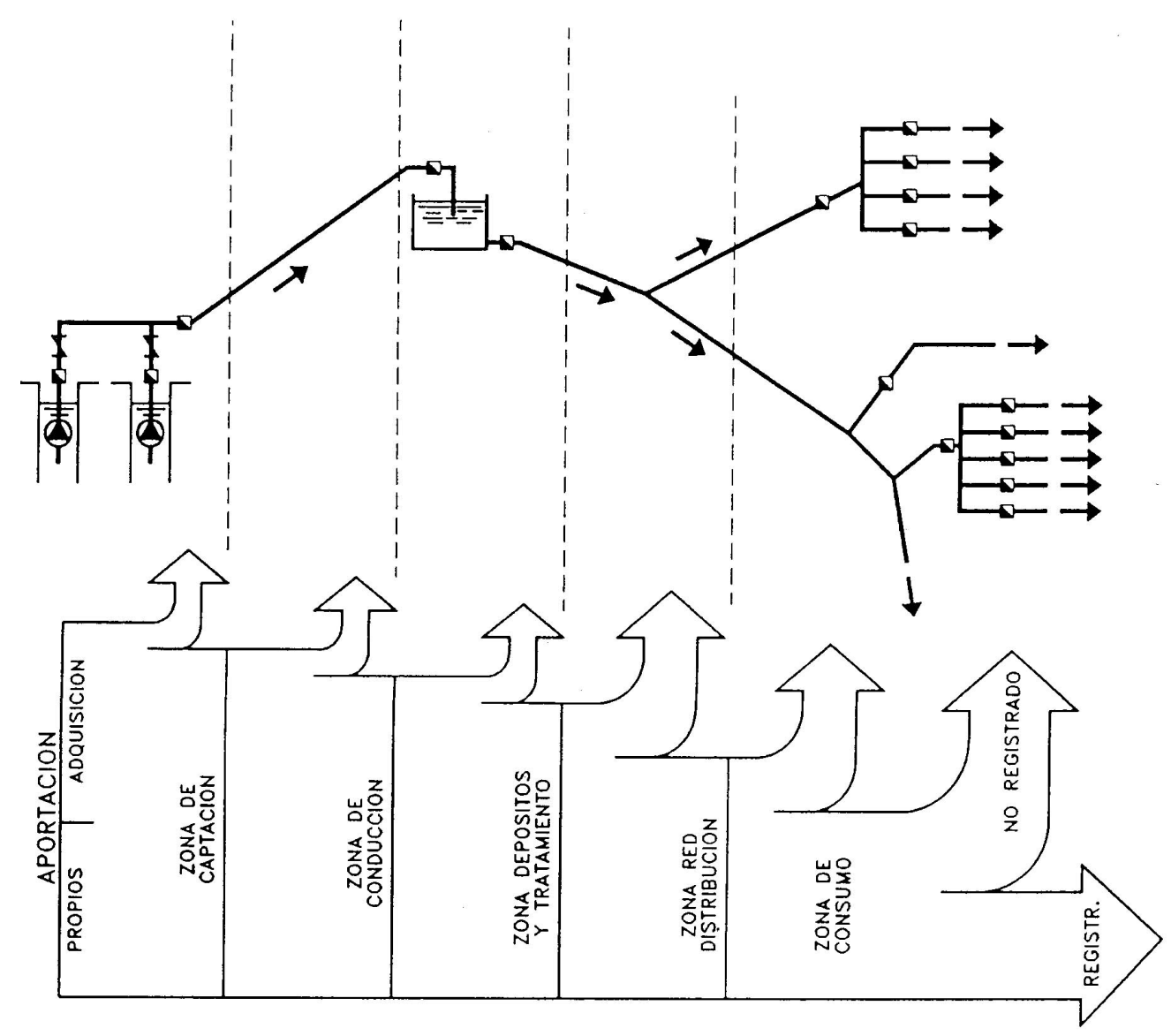

Figura 2. Distribución espacial de consumos no registrados en un abastecimiento de agua.

Este volumen no registrable, estaría constituido entre otros por:

\footnotetext{
- $\quad$ Las pérdidas de agua en roturas de elementos del sistema, principalmente tuberías.

- $\quad$ Las pérdidas de agua a través de defectos del sistema, que se denominan genéricamente fugas.

- $\quad$ Las pérdidas y evaporación en depósitos.

- Las extracciones clandestinas a través de conexiones ilegales.
}

Constituye la fracción del volumen no registrado generalmente más importante, por encima del $50 \%$.

\section{Concepto de rendimiento volumétrico}

Las pérdidas de agua, son causa de gran número de problemas económicos, higiénicos, técnicos y sociales. Aunque todo sistema hidráulico es vulnerable y por tanto sufre accidentes y fallos que producen entre otras consecuencias, pérdidas en el caudal transportado, el riesgo asumible debe estar claramente limitado.

La obsolescencia de los equipos y el deterioro progresivo de materiales e instalaciones provocan dichos fallos, que deben compensarse con un adecuado mantenimiento integral, y no reducirse sólo al imprescindible pero insuficiente mantenimiento correctivo.

Para efectuar un diagnóstico completo de un sistema real de distribución de agua conviene evaluar una serie de parámetros y determinar ciertos ratios que permitan conocer el estado del sistema y su eficacia. Indudablemente, todo buen diagnóstico exige un conocimiento detallado y preciso de los elementos que componen el sistema, de sus características y comportamiento, y también requiere un conjunto de mediciones de las variables de estado (presiones, caudales y potencias) en puntos estratégicos a lo largo del tiempo.

Uno de los ratios más importantes, entre los indicadores de la eficiencia del sistema, es sin duda el rendimiento volumétrico. El rendimiento volumétrico de una red o de un sector aislado de la misma, se define como la relación entre el volumen registrado y el volumen total aportado en un mismo periodo de referencia.

$$
\eta_{v}=\frac{V_{R E G}}{V_{\text {TOTAL }}}
$$


De acuerdo con el valor de dicho ratio se puede emitir un primer diagnóstico del estado de la red. En general, un valor superior al $15 \%$ puede considerarse aceptable.

\section{Otros ratios recomendables para efectuar un buen diagnóstico}

Obviamente, el rendimiento volumétrico medio no es, en general, suficiente para diagnosticar adecuadamente un sistema hidráulico, y por tanto dicho análisis debe completarse con la evaluación de otros ratios y parámetros de la explotación de dicho sistema. Asimismo es interesante analizar la evolución temporal de los mismos.

Un ratio representativo del estado de conservación de un sistema hidráulico es el volumen perdido por $\mathrm{Km}$. de tubería y día transcurrido. Este ratio tiene en cuenta la magnitud de la red pues en efecto conforme una red es más extensa es más vulnerable y por tanto su rendimiento volumétrico puede ser menor que en redes pequeñas para un mismo caudal circulante. Un valor guía orientativo puede ser 0,2 litros $/ \mathrm{seg} / \mathrm{Km}\left(17 \mathrm{~m}^{3}\right.$ $/ \mathrm{Km} / \mathrm{día})$.

Dado que el ratio anterior no tiene en cuenta la sección de las conducciones, resulta más apropiado usar alguno que incorpore el diámetro interior. El rango 0,2 - 0,3 $\mathrm{m}^{3} / \mathrm{km} / \mathrm{mm} /$ día, puede ser apto para redes de distribución de diámetro interior comprendido entre 60 y $150 \mathrm{~mm}$. Otro ratio interesante es el caudal unitario perdido en relación al número de acometidas en una zona o en un tramo; se expresa en litros/acometida/hora, y dependiendo de la separación entre acometidas y del estado de conservación del tramo, debe oscilar entre 3 y $101 / \mathrm{ac} / \mathrm{h}$.

Además de los ratios anteriores, también pueden citarse los siguientes para el diagnóstico técnico-económico de un abastecimiento de agua:

- Ratio de pérdidas económicas, en ptas globalmente perdidas por $\mathrm{Km}$ de tubería del sistema y año. Suele oscilar entre $100.000 \mathrm{y}$ $250.000 \mathrm{ptas} / \mathrm{Km} / \mathrm{año}$ en abastecimientos bien gestionados.

Ratio de pérdidas económicas, en ptas. por $\mathrm{m}^{3}$ de volumen aportado al sistema. Suele oscilar entre 4 y 8 ptas $/ \mathrm{m}^{3}$.

Gastos de inversión en reducción de pérdidas, en ptas por $\mathrm{Km}$ de tubería existente y año. Este ratio varía entre 8.000 y $15.000 \mathrm{ptas} / \mathrm{Km} / \mathrm{año}$.

Gastos de jnversión en reducción de pérdidas, en ptas por metro cúbico de agua aportada. Este valor varía entre 0,1 y $0,6 \mathrm{ptas} / \mathrm{m}^{3}$.

Porcentaje de pérdidas de agua por zonas o sectores de la red.
Porcentaje de pérdidas de agua sobre el total de agua inyectada, distinguiendo la posible causa de la pérdida (fugas latentes, roturas, errores de medición, ausencia de contador, evaporación, fraudes y consumos no controlados).

- $\quad$ Número de reparaciones por Km. de longitud de red y año

- $\quad$ Número de roturas por Km. de longitud de red y año

- Número de tugas detectadas por Km. de longitud de red y año

Un orden de magnitud de estos tres últimos ratios sería un valor comprendido entre 0,1 y 0,5 sucesos por $\mathrm{Km}$. y año. No obstante, aumenta como es lógico con la edad y vulnerabilidad de las redes.

Todos los valores anteriores proceden de estadísticas realizadas en España, y referenciadas en las publicaciones de ABAS (1990) y ABAS (1992).

\section{Situaciones indeseadas y condiciones inaceptables en un sistema de abastecimiento}

Un correcto diagnóstico de un sistema hidráulico a presión supone detectar situaciones y estados de funcionamiento de ese sistema que no satisfacen las necesidades previstas en su diseño o que efectivamente se requieren en el momento actual, así como evaluar los riesgos que comportan tales situaciones. Este análisis debe considerar tanto el funcionamiento en operación normal, como también en aquellas situaciones, accidentales o inesperadas, que pudieran producirse. En este sentido, el análisis no debe limitarse al régimen permanente, sino sobre todo debe ocuparse de los diferentes transitorios hidráulicos que se producen como consecuencia de la ocurrencia de sucesos, previstos o imprevistos, accidentales o provocados.

El análisis de los posibles fallos o malfuncionamiento de los elementos del sistema hidráulico, así como de sus causas y consecuencias, es fundamental tanto en el correcto diseño de dichos elementos y sus dispositivos de protección, como para el mantenimiento preventivo.

Sin entrar a enumerar todas las situaciones indeseadas que pueden presentarse en el funcionamiento de un sistema de abastecimiento y distribución de agua, pueden citarse:

a) Presiones inadecuadas, bien demasiado bajas o bien excesivas

b) Velocidades bajas o demasiado elevadas

c) Blujo inverso

d) Vibraciones perjudiciales

e) Interrupciones de suministro

f) Valores inapropiados en los parámetros de calidad 


\section{ESTRATEGIAS PARA MEJORAR EL APROVE- CHAMIENTO DEL AGUA CONSUMIDA Y REDUCIR LA DEMANDA GLOBAL DE AGUA}

Realizado el diagnóstico y el inventario preciso de las pérdidas y causas probables de las mismas, procede ahora decidir las estrategias a seguir y los métodos y técnicas a aplicar para su reducción o eliminación. No obstante, dado el carácter escaso y limitado del recurso hidráulico, actualmente acentuado por la persistente sequía en la mayor parte de nuestro país, parece también conveniente determinar primero qué estrategias serían las más adecuadas para reducir la demanda global de agua, tanto mediante la reducción de las pérdidas reales (responsabilidad de los gestores del abastecimiento), como ahorrando el volumen de agua que por despilfarro, descontrol o mal aprovechamiento en general, no es estrictamente necesario para cubrir las necesidades de la sociedad (responsabilidad de todos).

Si bien el presente artículo dedica mayor espacio al planteamiento y resolución del grave problema de las pérdidas reales de agua, no menos importante resulta el ahorro del agua efectivamente consumida. Por ello y en aras de un enfoque general del problema en su conjunto, se recogen también algunas políticas que permiten conseguir un ahorro en el consumo de agua por parte de los abonados y de todos los usuarios del sistema.

En primer lugar, debe insistirse en la importancia de conseguir mejorar el aprovechamiento del agua en todos y cada uno de los usos a que se destina, y ello no sólo en épocas de restricción, sino siempre, pues debe tratarse de un objetivo permanente. De hecho, el éxito o el fracaso de estas medidas reside en la persistencia de su aplicación y en la concienciación de toda la sociedad, ambas basadas en una política racional y debidamente justificada a toda la población.

Entre las estrategias que pueden emplearse se han escogido las siguientes:

1. Campañas de información y educación.

2. Políticas de persuasión de los usuarios para regular y reducir su consumo.

3. Racionamiento del volumen disponible (mediante porcentaje del uso normal).

4. Racionamiento del recurso (mediante limitación superior del consumo).

5. Restricciones de suministro (mediante interrupción durante un periodo).

6. Modificación de las acometidas e instalaciones de suministro.

7. Prohibición de derroches y despilfarras de consumo de agua.

8. Incorporación de contadores, facturando a precio constante el $\mathrm{m}^{3}$
9. Modificación de la tarifa, introduciendo bonificación por bajo consumo.

10. Introducción de una tarifa progresiva, estructurada por bloques de consumo.

11. Incorporación de una tarifa estacional para reducir más el consumo en verano.

12. Incentivos financieros para invertir en mejoras de las instalaciones interiores.

13. Penalizaciones económicas por el uso de instalaciones y aparatos poco eficientes.

Para tener una valoración orientativa del grado de aceptación de las mismas por parte de los consumidores (de $\mathrm{O}$ a 10 puntos), de su coste de implantación y de su eficacia relativa, se ha confeccionado la siguiente tablaresumen en base a las experiencias realizadas según Lord (1983), en tres ciudades medianas de Estados Unidos, de consumo predominantemente doméstico.

Tabla 1. Aceptabilidad y coste de diferentes políticas

\begin{tabular}{|c|c|c|}
\hline Estrategia & Aceptación & Coste \\
\hline 1 & 8,9 & Bajo \\
\hline 2 & 8,2 & Bajo \\
\hline 3 & 5,1 & Bajo \\
\hline 4 & 5,35 & Bajo \\
\hline 5 & 7,4 & Medio \\
\hline 6 & 7,1 & Alto \\
\hline 7 & 7,9 & Medio \\
\hline 8 & 7,0 & Medio \\
\hline 9 & 4,2 & Medio \\
\hline 10 & 6,35 & Medio \\
\hline 11 & 5,4 & Medio \\
\hline 12 & 7,93 & Alto \\
\hline 13 & 5,35 & Bajo \\
\hline
\end{tabular}

En cuanto a la eficacia real, las medidas de información $\mathrm{y}$ de persuasión son difíciles de cuantificar, pero su eficacia suele ser moderada (ahorros entre el 10 y el 20 $\%$, de volumen de agua registrado), siendo más efectivas las primeras que las segundas. Las medidas más efectivas resultan ser las basadas en la incorporación de contadores y modificación de la tarifa, que pueden suponer ahorros superiores al $30 \%$, dependiendo de la elasticidad de la demanda. Las demás medidas incentivas son en general de efectividad baja $o$ moderada, con ahorros en volumen por debajo del $17 \%$.

Como conclusión, parece lógico aplicar siempre una combinación de estrategias, incluyendo las de bajo coste, y sobre todo las que repercuten en la tarifa, 
recomendando la instalación de contadores y la implantación de precios crecientes con el consumo. La efectividad de una campaña múltiple será generalmente mayor y además ésta se mantendrá más en el tiempo.

\section{CARACTERIZACIÓN Y ESTIMACIÓN DE LA EFICIENCIA DE LAS ESTRATEGIAS DE LO- CALIZACION DE FUGAS}

Dado que la gran parte del volumen perdido es consecuencia de los defectos, provocados o accidentales, conviene analizar su distribución e importancia relativa, para preseleccionar las estrategias. Por otro lado, es necesario determinar las características técnicas básicas de las mismas, y estimar su efectividad real en la detección y localización de fugas en una red hidráulica.

Los conceptos fundamentales que permiten caracterizar una estrategia de localización de fugas son, entre otros, los siguientes:

Ámbito de aplicación. Tipo de instalaciones que pueden ser revisadas con esa estrategia, con una garantía mínima.

Rango de detección. Delimitación del tipo y magnitud de defectos que pueden ser detectados y localizados cuando se aplica correctamente esa estrategia, con una probabilidad aceptable.

Eficacia del método. Se podría definir como la fracción de defectos existentes, no considerados latentes, que son detectados en promedio en instalaciones revisadas con esa estrategia, bajo condiciones normales y con personal debidamente cualificado y experimentado.

Eficiencia volumétrica. Se define como la fracción del volumen perdido en todos los defectos existentes en la instalación auditada, que será ahorrado como consecuencia de la posterior reparación de los defectos localizados con esa estrategia.

Las características intrínsecas de cada método suelen estar muy condicionadas por la aplicación concreta del mismo a cada instalación particular por parte de los equipos de técnicos encargados, es decir, factores externos como la preparación y experiencia del personal encargado pueden tener una influencia decisiva.

Con independencia de lo anterior, debe considerarse la trascendencia que tiene, en la eficacia y eficiencia de una estrategia, la distribución de los defectos en cuanto a su magnitud. Con frecuencia este aspecto fundamental es ignorado, siendo que esa distribución almacena una información crucial para la estimación de la eficiencia técnica y económica. Si se ordenaran los defectos existentes, de mayor magnitud a menor, y se procediera al análisis de la distribución resultante, podría obtenerse una curva de forma similar a la que muestra la Figura 3, y que puede aproximarse a una exponencial decreciente con el número de fugas. Esta conclusión semiempírica, ha sido avalada experimentalmente efectuando un análisis estadístico de tres ciudades españolas.

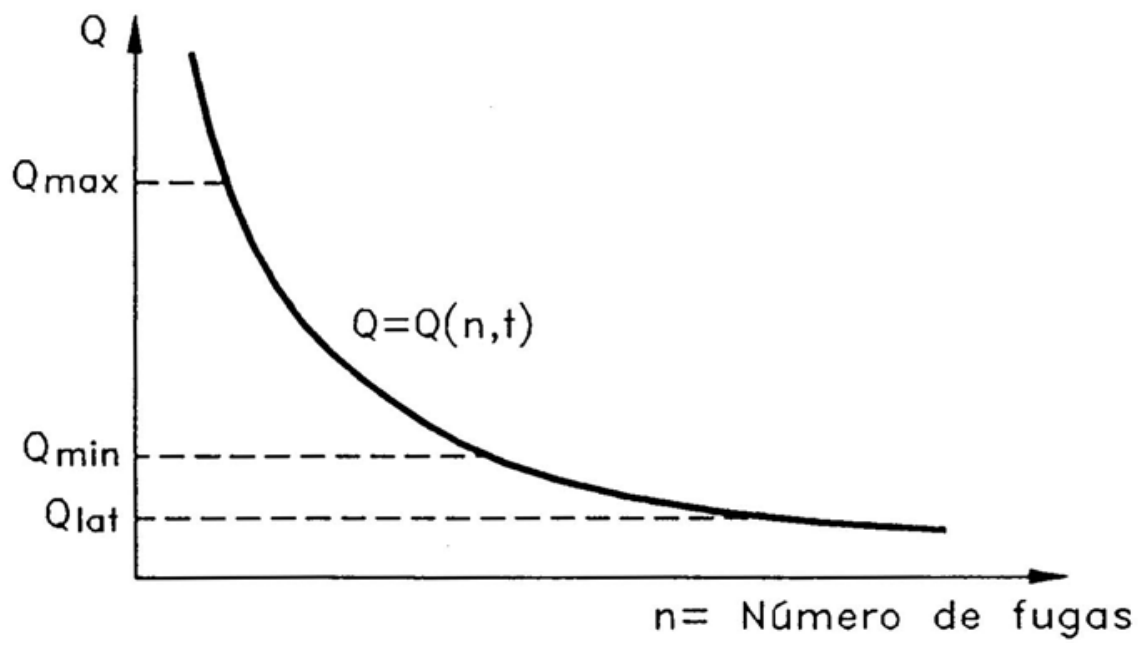

Figura 3 Distribución genérica de las fugas en una red, clasificadas por caudales.

Asumiendo por tanto, una expresión analítica del caudal de fugas según la ordenación anterior de la forma:

$$
Q(n)=Q_{0} e^{-b-n}
$$

donde $\mathrm{Q}(\mathrm{n})$ es el caudal perdido en la fuga número n, y Qo y b son dos parámetros del ajuste exponencial efectuado.

Se podrían estimar la eficacia y la eficiencia del método de detección, considerando como rango de aplicación del mismo el intervalo de caudales de fuga 
(Qmín’ Qmáx) que corresponden respectivamente al caudal umbral de detección y al caudal máximo que se detecta o que simplemente al añorar a la superficie es visualmente localizado. Si denominamos por $\mathrm{Q}_{\text {lat }}$ al caudal límite superior de las fugas consideradas como latentes, las fugas cuya magnitud sea inferior a $\mathrm{Q}_{\text {lat }}$ constituirá un conjunto de pequeña importancia técnica y económica, siendo su presencia perfectamente asumible, de modo que tendremos:

$$
\begin{gathered}
\text { Eficacia }=\frac{n\left(Q_{\text {mín }}\right)-n\left(Q_{\text {máx }}\right)}{n\left(Q_{\text {lat }}\right)} \\
=\frac{\operatorname{In}\left(Q_{\text {máx }} / Q_{\text {mín }}\right)}{\operatorname{In}\left(Q_{0} / Q_{\text {lat }}\right)} \\
\text { Eficacia }=\frac{\left(Q_{\text {máx }}-Q_{\text {mín }}\right)}{Q_{\text {máx }}}
\end{gathered}
$$

Las expresiones anteriores, pueden deducirse sin dificultad a partir de las definiciones respectivas y la expresión (4). En cualquier caso su valor se verá afectado en función de la influencia de los factores externos, del método concreto y del tipo de instalación, resultando finalmente unos valores prácticos menores.

En vista de la peculiar forma que presenta la curva y los valores de la eficiencia que se obtienen a partir de ella, se puede observar que una pequeña fracción de fallos es la responsable de la mayor parte del caudal total perdido, lo que recomienda actuar selectivamente aprovechando este hecho. De la experiencia real se deducen valores orientativos, que estiman que un $25 \%$ de los defectos pierden más del $70 \%$ del volumen global de fugas.

\section{SELECCIÓN DE ESTRATEGIAS PARA REDUCIR EL VOLUMEN DE PERDIDAS Y OPTI- MIZACION DE LAS MISMAS}

La reducción global de pérdidas de agua requiere de la aplicación de una serie de métodos, procedimientos, técnicas y modificaciones que requieren tanto de más inversiones puntuales como del mantenimiento de unos costes de explotación mientras dura su aplicación. Por otro lado, como consecuencia de su puesta en marcha, se obtienen beneficios económicos y una mayor eficiencia técnica, fruto de esas mejoras y del ahorro de agua que deben justificar las inversiones realizadas.

La selección correcta entre las posible alternativas no debe hacerse exclusivamente en base a criterios económicos de rentabilidad; dicho criterio es sólo un poderoso instrumento de ayuda a la toma final de decisiones, pero han de tenerse presentes otros criterios supraeconómicos y técnicos, como el de garantizar una calidad de suministro mínima (cumplimiento de unas especificaciones técnicas y obtención de una eficiencia aceptable), y el de reducir el riesgo. Estos aspectos se incorporarán como restricciones en el problema de selección y optimización de las diferentes estrategias rentables, según el criterio económico utilizado.

En total concordancia con las tendencias actuales que integran todas las estrategias dirigidas a conseguir que el sistema de abastecimiento alcance un estado óptimo de operación y se mantenga con cierto grado de seguridad en el entorno del mismo, se propone a continuación un método para analizar y seleccionar estas estrategias.

En este método no sólo se jerarquizan las inversiones entre sí, sino que se evalúan sus respectivas rentabilidades. Los datos que se precisan para confeccionar el modelo de son dos :

\section{$I=\quad$ Montante de la inversión (en pías.) \\ $B=$ Beneficios diferenciales de efectuar o no la inversión.}

Así como el dato correspondiente a la inversión necesaria es relativamente fácil de obtener, calculándolo o estimándolo, los beneficios están compuestos por una serie de sumandos que son afectados por la incertidumbre propia del problema y son de carácter aleatorio.

Los beneficios diferenciales se componen de los siguientes sumandos:

a) Disminución de riesgo conseguida con la realización de la inversión

Es sin duda el término más difícil de cuantificar monetariamente, pero también es el más importante y el que justifica básicamente la decisión de hacer o no una inversión que permita implantar una determinada estrategia.

Dado que se trata de una inversión para aumentar la seguridad, debe definirse adecuadamente el riesgo que supone el funcionamiento del sistema, con y $\sin$ la inversión. El riesgo se puede definir como la incertidumbre de ocurrencia de unas pérdidas y daños en general, consecuencia de un suceso-fallo o del deterioro del sistema y de la magnitud de éstos en tal caso. La magnitud del riesgo es difícil calcularla, por su propio carácter intrínseco. Una expresión generalmente aceptada es la siguiente:

$\mathfrak{R}=$ Probabilidad $\bullet$ Consecuencia $\bullet$ Duración

Obviamente, las consecuencias se valoran en ptas por unidad de tiempo de duración del suceso. La inversión que se está evaluando puede disminuir el riesgo de varias formas: 
- Disminuyendo la probabilidad de ocurrencia (aumento de fiabilidad del sistema).

- $\quad$ Reduciendo la magnitud del daño o de sus consecuencias.

Disminuyendo el periodo de tiempo de duración del estado anómalo.

También puede haber inversiones que consigan reducir los tres factores simultáneamente, lo que supone una mayor reducción del riesgo.

Este primer término del beneficio diferencial equivale por tanto a la diferencia entre riesgos, sin y con inversión:

$$
\mathbf{B}_{1}=\mathfrak{R}_{0}-\mathfrak{R}_{\mathbf{i}}
$$

\section{b) Reducción de gastos de explotación del abastecimiento}

La reducción en las pérdidas de agua conlleva entre otros beneficios:

- $\quad$ la reducción de caudales, circulantes, lo que por un lado disminuye considerablemente las pérdidas de carga en las conducciones (aproximadamente proporcionales al cuadrado del caudal) y en consecuencia puede reducirse la presión necesaria en los puntos de inyección de la red de distribución. En definitiva se requiere una menor potencia en cabecera y se produce un ahorro energético.

- $\quad$ la reducción en el volumen total aportado reporta también otro tipo de beneficios, como son la reducción en productos de tratamiento y desinfectantes.

- $\quad$ en teoría, y dado que el sistema habrá sido diseñado correctamente, el estado de funcionamiento será más cercano al preVISTO EN DISEÑO, Y EN CONSECUENcia más próximo al óptimo. En este sentido se mejorará su eficacia y eficiencia globales, y se alargará su vida útil.

Los beneficios anteriores deben ser evaluados económicamente, y en dicha previsión deberá tenerse en cuenta que el coste marginal de captación, almacenamiento, tratamiento y distribución de las unidades de volumen ahorradas es mayor que el coste medio, puesto que se consideran como si fueran las últimas en ser producidas. Así, por ejemplo, si se está consumiendo energía eléctrica en horas punta para satisfacer la demanda, y mediante la aplicación de una serie de medidas (programadas según cierta estrategia) es posible reducir la producción hasta el extremo de no precisar bombear en horas punta, el ahorro económico es aún mayor.

La forma de evaluar este ahorro será por diferencia entre gastos de explotación, antes y después de la inversión:

$$
\mathbf{B}_{\mathbf{2}}=\mathbf{G E}_{\mathbf{0}}-\mathbf{G E}_{\mathrm{i}}
$$

c) Reducción en los costes globales de
mantenimiento

En este caso, debe contabilizarse la diferencia en los costes de ambos programas globales de mantenimiento, sin efectuar la inversión i, y realizando tal inversión. En muchas ocasiones este balance resulta negativo, debido a una mayor frecuencia de las reparaciones y a un mayor coste de inspecciones y comprobaciones de los elementos del sistema de abastecimiento; ello no debe extrañar y es parte del precio que hay que pagar para restituir al sistema a un estado aceptable y mantenerlo en él.

$$
\mathbf{B}_{3}=\mathbf{C M}_{0}-\mathbf{C M}_{\mathbf{i}}
$$

\section{d) Aplazamiento de otras inversiones necesarias y otros beneficios.}

Finalmente, y con el objetivo de no dejar fuera ningún concepto importante en este análisis, conviene también incluir el indudable beneficio que supone retrasar ciertas inversiones de ampliación y rediseño del sistema de abastecimiento, como consecuencia de haber frenado el crecimiento de la demanda. Además, puede ocurrir incluso, que ya no resulte necesario realizar esas inversiones, caso frecuente en abastecimientos de población estabilizada y con dotación prácticamente constante.

La contabilización rigurosa de este retraso en las inversiones, disminución de su montante o su posible eliminación, requiere de una actualización del valor económico según sea la tasa de interés equivalente $r^{\prime}$, corrigiendo la tasa de interés real $r$ por el efecto de la tasa de inflación s, según la expresión:

$$
1+\mathrm{r}^{\prime}=\frac{1+r}{l+s} \quad ; \quad r^{\prime} \frac{r-s}{l+s}
$$

Si las inversiones previstas inicialmente $\mathrm{lo}_{2}, \mathrm{Io}_{2}, \ldots$, iban a realizarse en los años $\mathrm{k},, \mathrm{k}, \ldots, \ldots, \mathrm{k}$, respectivamente y ahora serán retrasadas a los años $\mathrm{m}, \mathrm{m}_{2}, \ldots, \mathrm{m}_{\mathrm{i}}$, pudiéndose reducir sus valores o anularse, siendo éstos entonces $\mathrm{I}_{1}, \mathrm{I}_{2} \ldots, \mathrm{I}_{3}$,el ahorro actualizado al año actual será:

$$
B_{4, a c t}=\sum_{j}\left(\frac{I o_{j}}{\left(1+r^{\prime}\right)^{k j}}-\frac{I_{j}}{\left(1+r^{\prime}\right)^{m j}}\right)
$$

Análogamente a como se ha procedido con las inversiones previstas o planificadas, podrían incorporarse también las indemnizaciones y penalizaciones por daños a terceros, según el entorno legislativo, y que no son despreciables en muchos servicios de agua 
En resumen, mediante la suma de todos los términos anteriores se puede calcular el beneficio diferencial, o beneficio neto, correspondiente a cada una de las estrategias candidatas. Con los valores anteriores pueden calcularse los ratios económico-financieros, determinando con ello la rentabilidad de cada opción y estableciendo una jerarquía en la serie de estrategias que hayan resultado rentables.

Entre los métodos de análisis de rentabilidad debe optarse por usar aquellos que actualizan el valor del dinero, y además de manera continua. En realidad, los beneficios diferenciales $\mathbf{B}$ no se generan al final de cada año, sino a lo largo del mismo según una distribución continua.

Para evaluar la rentabilidad de cada estrategia concreta se calculará el valor capital VC, de la inversión I, según la expresión discreta:

$$
V C=-I+\sum_{j=1}^{n} \frac{B_{j}}{\left(1+r^{\prime}\right)^{j}}
$$

donde $\mathrm{n}$ representa el periodo de aplicación de la estrategia, en años. Sin embargo, es mejor según la expresión continua:

$$
V C=-I \int_{t=0}^{t=n} \frac{B(t) d t}{\left(1+r^{\prime}(t)\right)^{t}}
$$

El criterio de selección de estrategias consiste en aceptar aquellas cuyo valor capital sea positivo, y además permite establecer con rigor una jerarquía de las mismas, siendo la más rentable aquella que tenga el mayor valor capital.

Alternativamente puede realizarse la selección en base al criterio de rentabilidad propia de cada estrategia, que además permitiría compararla con la mínima deseada o aceptable. Para obtener la rentabilidad -propia- interna, $\mathbf{r}_{\mathbf{p}}$, bastará con determinar el valor de la misma que anula el valor actual, $\mathrm{VC}=\mathrm{O}$, resolviendo la ecuación correspondiente. La tasa interna de rentabilidad, así calculada, permite decidir qué estrategias deben ser aplicadas, y el orden de preferencia en el conjunto de todas las inversiones planteadas, además de las encaminadas a reducir pérdidas.

El modelo anterior sirve para comparar alternativas diferentes y asignar prioridades sobre el uso del dinero (que al igual que el agua es un recurso escaso y presenta un coste de oportunidad), para determinar los métodos más eficientes en la mejora del sistema, en general, y de la reducción de pérdidas, en particular. Otros procedimientos aproximados para analizar la rentabilidad, como el retorno sobre inversión (ROÍ) y el plazo de retorno ("pay back") no son recomendables, pues no actualizan adecuadamente el valor del dinero.

\section{OPTIMIZACION DE ESTRATEGIAS PARA LA MEJORA DE UN ABASTECIMIENTO}

Establecida la jerarquía de rentabilidad de las estrategias, procede ahora plantear globalmente la optimiza-ción conjunta de todas las que vayan a ser adoptadas finalmente. En primer lugar convendría insistir en la enorme importancia que tiene coordinar dichas estrategias, determinar el orden, instante de inicio y ámbito de aplicación; para ello debe resolverse un problema de optimización.

Tradicionalmente se ha optado por aplicar las estrategias más rentables y realizar las inversiones pertinentes, con los únicos criterios de funcionalidad y de rentabilidad económica. Actualmente es conveniente exigir además unas restricciones adicionales. No es suficiente con que el sistema funcione y su explotación sea rentable, sino que lo haga con una eficacia, eficiencia y seguridad mínimas.

Las restricciones anteriores se traducen en el establecimiento de un criterio técnico que determine unos valores mínimos en los ratios indicadores de la bondad del sistema, como el rendimiento volumétrico o el caudal perdido por km y año.

Obviamente, para compensar esa mayor bondad y calidad de suministro deberán efectuarse más inversiones y aplicar nuevas estrategias, lo que aumentará los gastos y exigirá una compensación en los ingresos. Sin embargo, ello estará perfectamente justificado, al haber alcanzado no sólo un estado aceptable, sino mantenerse en él aprovechando mucho mejor los escasos y limitados recursos de capital, hídricos y energéticos, entre otros.

El conjunto de usuarios de un sistema de abastecimiento, y la sociedad en general, deberán exigir un servicio cada vez mejor gestionado y de mayor calidad, lo que justificará un aumento en su aportación económica conjunta para mantenerlo en ese nuevo estado.

Estos objetivos deberían hacer reflexionar sobre la importancia de mejorar la eficiencia volumétrica y energética de los sistemas hidráulicos existentes antes de proceder a su ampliación o a la búsqueda de nuevas fuentes de recursos, aunque estas soluciones fueran más interesantes económicamente a corto plazo.

Así pues, el planteamiento general del problema de optimización de un abastecimiento debería componerse de una función objetivo a minimizar, sujeta a unas ciertas restricciones como a continuación se expresa:

\section{OBJETIVO:}

min (Coste global del Programa de Mejora) 


\section{RESTRICCIONES:}

a) Especificaciones técnicas :

$$
\left(\mathrm{Q}_{\mathrm{u}, \mathrm{i}}\left(\mathrm{t}, \mathrm{p}_{\mathrm{i}}\right) ; \mathrm{p}_{\mathrm{i}}\right) \geq \text { Valores mínimos }
$$

b) $\quad$ Riesgo $=\mathfrak{R}(\mathrm{t}) \leq \Re$ máximo aceptable

donde :

i se refiere a una acometida genérica o a una agrupación de ellas.

t es el instante de tiempo

$\mathbf{p}_{\mathbf{i}}$ es la presión relativa en la acometida $\mathrm{i}$, que deberá superar un mínimo.

$\mathbf{Q}_{\mathbf{u}, \mathbf{i}}$ es el caudal útil suministrable a través de i, que deberá superar el mínimo necesario.

De esta forma, aunque se sigue pretendiendo disminuir los costes relativos a la implantación del programa, suma de la amortización anual de las inversiones, de los gastos anuales de su aplicación y el valor económico de las reducción de volumen registrado, se exige al mismo tiempo un funcionamiento aceptable y con un riesgo acotado superiormente.

Dada la dificultad en cuantificar las restricciones, se puede exigir el cumplimiento de ciertos requisitos basados en rangos de valores de las variables hidráulicos (por ejemplo que las presiones se encuentren dentro de un intervalo), en parámetros indicadores de la eficiencia (como exigir un rendimiento volumétrico mínimo) y en la fiabilidad (limitar el número de interrupciones de suministro y roturas por unidad de longitud y tiempo).

Como consecuencia de la resolución de este problema, se determinarán los instantes de inicio de aplicación de cada estrategia preseleccionada (por eficiencia y rentabilidad) y los elementos o longitudes de conducción afectadas, obteniéndose también la magnitud de las inversiones a realizar. Finalmente, se comprobará el cumplimiento de las restricciones funcionales, de rendimiento y de seguridad, y en caso contrario deberá equilibrarse de nuevo el balance económico entre gastos e ingresos necesarios para conseguirlo.

Actualmente, muchos sistemas hidráulicos de abastecimiento y sobre todo los de riego agrícola presentan rendimientos muy bajos, incluso por debajo del $50 \%$, y sin embargo su explotación es rentable, dado que el coste marginal de producción es cercano al coste medio, en cualquier caso bajo, y por otro lado la disponibilidad del recurso no presenta serios problemas en esos lugares. Esta gestión no es plausible en otros lugares, muy próximos incluso, al no ser solidaria con la escasez del agua lo que exige un profundo cambio cualitativo.

\section{PROBLEMAS DERIVADOS DE LA INSUFICIENCIA O DEL EXCESO DE PRESIÓN}

En realidad, buena parte de los problemas que se presentan con frecuencia en los abastecimientos de agua son consecuencia, directa o indirecta, del estado de presiones existente y de su variación temporal.

Entre los problemas generados por las situaciones de baja presión destacan:

- Impedir o dificultar a ciertos usuarios satisfacer adecuadamente su demanda

- $\quad$ la ocurrencia de cavitación

- $\quad$ intrusiones de aire y líquidos contaminantes del entorno en la red.

- $\quad$ roturas por aplastamiento

Conviene además en todo abastecimiento limitar también las presiones superiormente, estableciendo por tanto un rango recomendable de las presiones en cada punto del sistema.

Más que el hecho, en sí ya preocupante, de la existencia de presiones muy altas, lo que resulta verdaderamente arriesgado es permitir variaciones bruscas de la presión. Para analizar este problema, deben conocerse los límites de trabajo, sobre todo en cuanto a presiones y variaciones de la misma, de todos los elementos que constituyen el sistema hidráulico, en concreto tuberías, juntas, válvulas, elementos de protección y regulación, y también la instrumentación. Uno de los objetivos que se pretenden en la explotación de un abastecimiento es, sin duda, impedir que los estados de funcionamiento de los elementos se encuentren fuera del rango comprendido entre los límites recomendados, tanto en operación normal como durante los transitorios hidráulicos.

Entre los principales problemas que se presentan o que puedan aparecer como consecuencia de presiones elevadas destacan:

- Incremento de los caudales perdidos a través de los defectos existentes

- $\quad$ Aumento de la probabilidad de generación de nuevos defectos o aumento de la magnitud de los ya existentes.

Incremento en la probabilidad de producción de roturas en los elementos de conducción, accesorios y en general de avería en todos los demás.

- $\quad$ Aparición de ruidos y vibraciones, o aumento en la magnitud de éstos.

Aumento del riesgo de sobrepresiones ocasionadas por bolsas de aire acumuladas en el interior de las conducciones. 
Es sumamente interesante modelizar el comportamiento de la red teniendo en cuenta la dependencia entre presión y caudal demandado en cada nudo. Además, es notorio y ha sido comprobado experimentalmente que el volumen perdido en fugas tiende a aumentar con el tiempo más que linealmente con la presión media, lo que exige un control estricto de las presiones existentes. La probabilidad de rotura aumenta con la brusquedad de los cambios experimentados en la presión y con la magnitud de ésta, como se muestra en la Figura 4, extraída de Vela (1993).

\section{MEDIDAS A CONSIDERAR PARA REDUCIR PERDIDAS DE AGUA EN UNA RED Y RECOMENDACIONES RESPECTO DE SU EFECTIVIDAD}

La finalidad de TODAS estas medidas se resumiría en la reducción del riesgo, entendiendo por éste, como ya se indicó anteriormente en el epígrafe Optimización de Estrategias para Mejorar un Abastecimiento, el producto de la probabilidad de ocurrir un suceso (avería, fallo o rotura), por la magnitud del daño ocasionable (volumen de agua perdido, coste de reparación, etc..) y

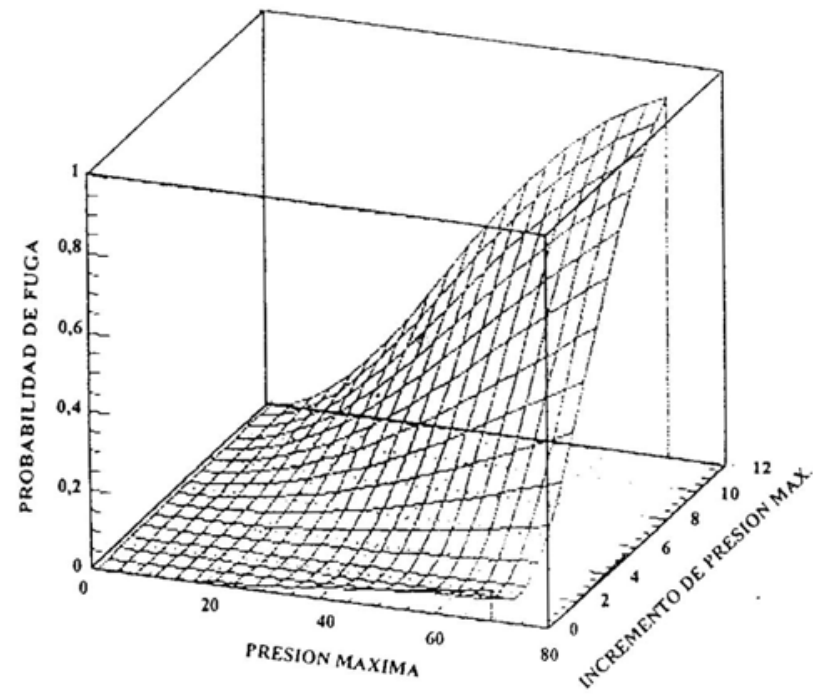

por el tiempo transcurrido hasta la restitución al estado normal.

\section{Clasificación de las medidas existentes para reducir pérdidas de agua}

En el sentido anterior, dichas medidas se podrían clasificar en:

\section{Medidas preventivas}

Aquellas encaminadas a reducir la probabilidad de ocurrencia de los sucesos indeseables, o de situaciones anómalas.

\section{Medidas preventivas-correctivas}

Aquellas dirigidas a reducir la probabilidad de ocurrencia de los fallos y además, en caso de producirse esos sucesos indeseados, a reducir la magnitud del daño producido o el tiempo transcurrido hasta la restauración de las condiciones normales.

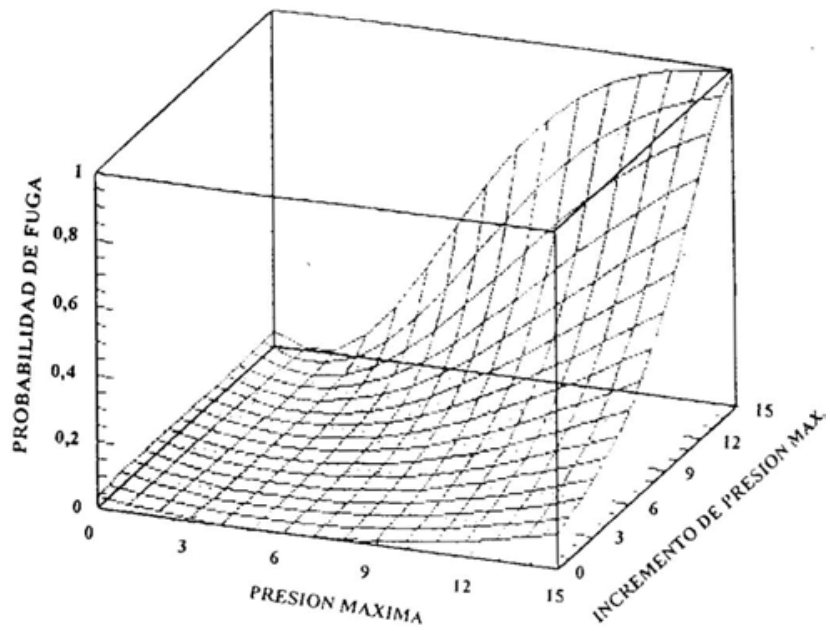

Figura 4. Influencia de la presión y su variación en la probabilidad de roturan en una red de agua.

\section{Medidas correctivas}

Aquellas encaminadas a reducir exclusivamente la magnitud del daño producido o el tiempo transcurrido hasta la restauración de las condiciones normales. Básicamente, consisten en la detección, localización y posterior reparación de los fallos y defectos existentes.

Entre las medidas anteriores pueden citarse por su importancia las siguientes:

\section{Preventivas:}

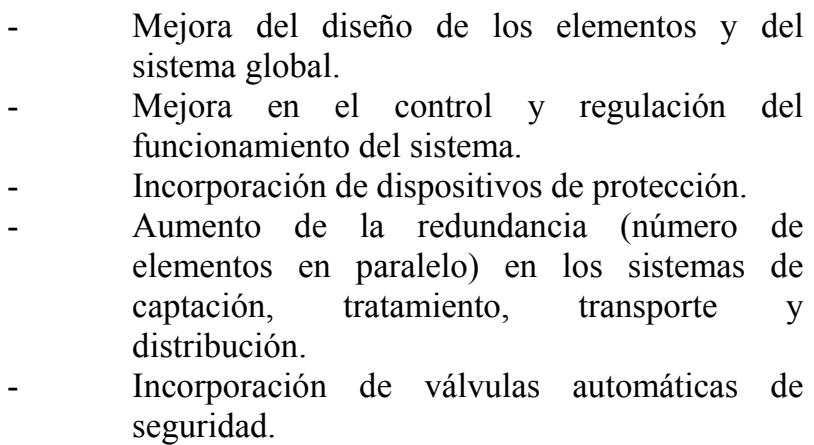


Mejora del diagnóstico de los sistemas mediante análisis y simulación del comportamiento de los sistemas de abastecimiento en todas las situaciones, con modelos matemáticos adecuados para tales fines, incluyendo el aspecto de fiabilidad.

\section{Preventi vas-correctivas:}

Zonificación y posterior sectorización de la red.

Planificación adecuada de las posibles rehabilitaciones, reposiciones o sustituciones de todos los elementos, determinando la secuencia adecuada de operaciones y los instantes óptimos para llevarlas a cabo.

Control de las presiones mediante válvulas reducto-ras de presión o válvulas reguladoras.

Homogeneización de presiones.

Reducción de presiones de inyección.

Selección y comprobación de estas medidas mediante el uso de modelos matemáticos.

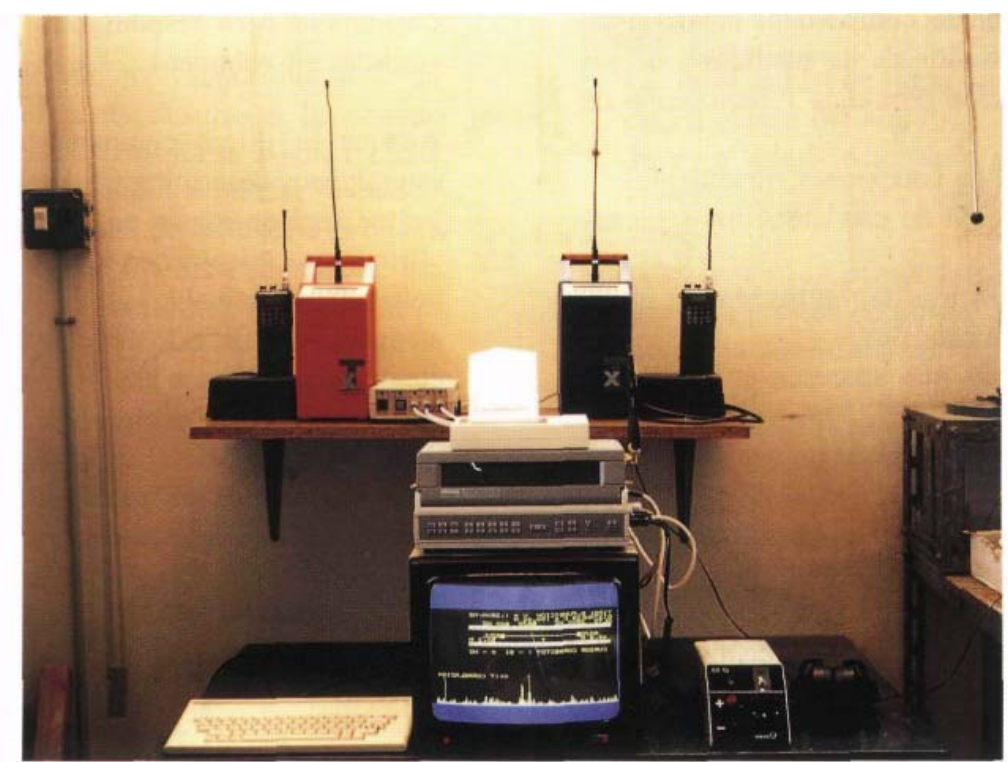

Fotografía 1. Equipo e instrumental auxiliar de detección de fugas por correlación acústica (gentileza de Aguas de Valencia S.A.)

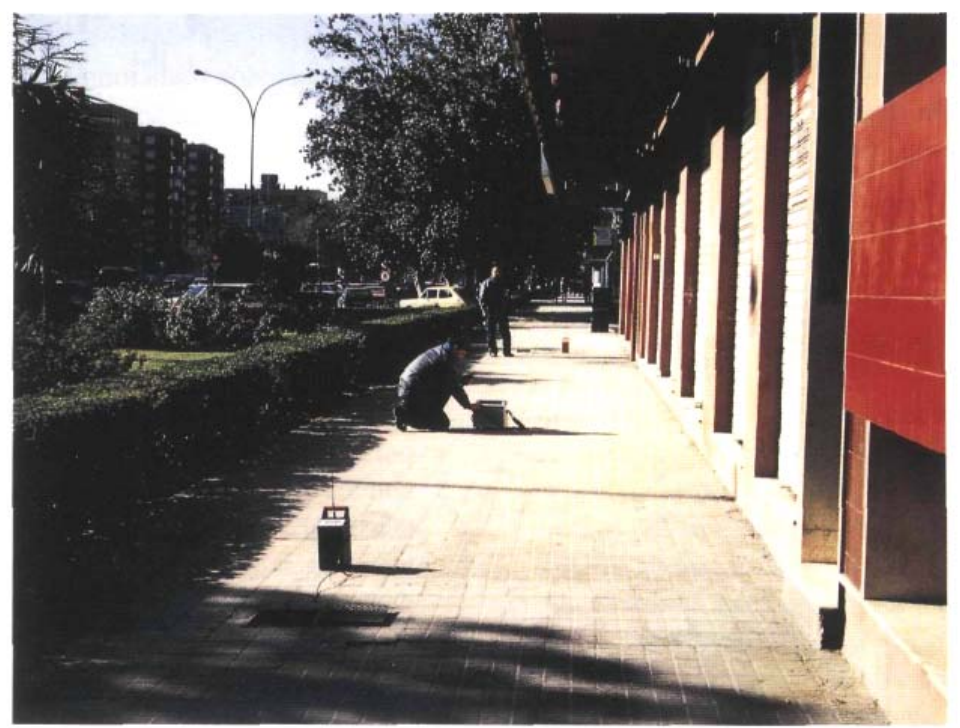

Fotografía 2. Detección práctica de fugas en la acera mediante correlación acústica (gentileza de Aguas de Valencia S.A.) 


\section{Correctivas:}

- $\quad$ Análisis y control periódicos de las pérdidas de agua

- Incorporación de válvulas de seguridad contra fugas y roturas.

- Mejora de los métodos de detección y localización de fugas.

- $\quad$ Aumento de la rapidez, precisión y capacidad de detección-localización de defectos mediante el concurso de un modelo calibrado por el método inverso (Ligget, 1992; Vela, 1993).

- Seguimiento continuo del comportamiento del sistema, mediante supervisión de las medidas y de sus variaciones, inspección periódica y realización de pruebas.

- $\quad$ Búsqueda y control de conexiones fraudulentas.

- Detección y reparación de caudalímetros y contadores defectuosos.

Confección de estadísticas de roturas según causas.

- Control nocturno de caudales.

- $\quad$ Análisis de las aguas que aparecen en sótanos y otros lugares.

- Control de las redes de saneamiento y de los caudales transportados.

- Control activo de escuchas, con correlador, geófo-nos, hidrófonos y resto de equipos.

Inspección de instalaciones interiores.

- Detección mediante análisis químico de muestras de agua y uso de trazadores, preferentemente conservativos (por ejemplo compuestos de flúor).

Recomendaciones respecto de la estrategia general a seguir en la selección y aplicación de las medidas para reducir pérdidas de agua de manera efectiva

En base a la propia experiencia de los autores en este área y la información analizada procedente de otros investigadores se puede recomendar una estrategia general, en cualquier caso flexible y modificable para cada abastecimiento en particular, que sirva para reducir las pérdidas de manera eficiente.

El procedimiento que se recomienda consiste en :

1. Realizar un buen diagnóstico del abastecimiento (ver Diagnóstico de un Sistema Hidráulico a Presión)

2. Zonificar y sectorizar el sistema. Jerarquizar los sectores según su eficiencia.

3. Jerarquizar las estrategias aplicables a los sectores (ver Caracterización y Estimación de la Eficiencia de Estrategias de Localización de Fugas y Selección de Estrategias para reducir el Volumen de Pérdidas $v$ Optimización de las mismas)

4. Optimización conjunta de las estrategias elegidas, con la ayuda de un modelo matemático, (ver epígrafes Optimización de
Estrategias para Mejorar un Abastecimiento e Influencia de la Modulación de la Demanda en el Volumen de Fugas y en las Prestaciones de una Red).

5. Proceder a implantar la estrategia en un sector determinado, como "experiencia piloto".

6. Extensión a los restantes sectores que lo requieran.

Normalmente se recomienda emplear siempre métodos preventivos, y respecto de los correctivos se aconseja aplicarlos selectivamente en los sectores con más pérdidas, y empleando técnicas eficientes que detecten con mayor probabilidad los grandes defectos (menores en número pero responsables de la mayoría de las pérdidas en volumen).

Aunque es difícil comparar a priori las diferentes estrategias y desarrollar en detalle las más.importantes lo que será objeto de un artículo posterior, se puede afirmar que mientras el ratio fugas/Km/año sea bajo, no resultando rentable la renovación parcial de algunos elementos (Andrés, 1992) conviene sectorizar la red y controlar periódicamente presiones y caudales, procediendo primero a prelocalizar aproximadamente los defectos importantes (mediante el método inverso en base a un modelo matemático), y localizándolos finalmente mediante correlación acústica.

\section{UTILIZACIÓN DE MODELOS MATEMÁTICOS PARA LA SIMULACIÓN Y LOCALIZACIÓN DE FUGAS}

El análisis y resolución del problema de pérdidas de agua y otros que presentan los sistemas hidráulicos exige actualmente el concurso de la modelización matemática de dichos sistemas, incorporando también los defectos.

\section{Modelización de las fugas de una red}

En los métodos tradicionales se admite la hipótesis de rendimiento volumétrico uniforme en toda la red. Este procedimiento es aproximado y supone proporcionalidad entre caudales de fuga y caudales controlados. En realidad, el rendimiento varía a lo largo del tiempo, y toma valores distintos en unas zonas u otras de la misma red.

Un método más correcto para la estimación de las cargas debería independizar los caudales demandados por los usuarios de las fugas, evaluando los primeros con los criterios pertinentes (facturaciones, dotaciones, densidad de población, ubicación de acometidas, etc..) y los caudales de pérdidas en función de los defectos de la red y del valor de la presión en las inmediaciones de los mismos.

En esencia el método consiste en diferenciar dos tipos de nudos de consumo en el modelo matemático del sistema de distribución: los denominados nudos de consumo 
útil, y los nudos de consumo no controlado. Los primeros son reales desde el punto de vista del esqueleto de la red, mientras los segundos son ficticios.

El procedimiento que se presenta trata por separado el caudal útil del que no lo es, distribuyendo el primero entre los nudos reales según un criterio convencional de reparto, basado en las facturaciones por ejemplo. El resto de caudal es demandado por el otro conjunto de nudos, según el estado de presiones. Este conjunto de nudos "ficticios" representa los defectos existentes en la red, causantes de las fugas.

Cada nudo real del modelo representa una zona de influencia del mismo, en la que la suma de las demandas útiles pertenecientes a la misma se asigna a dicho nudo en cada instante de tiempo; simultáneamente se pierde un caudal en dicha zona de influencia debido a la existencia de defectos en las instalaciones de esa área. A efectos e cálculo asumiremos como hipótesis simplificativa que se puede simular dicho escape con una válvula de descarga libre, cuyo coeficiente de descarga sea conocido. Mientras en los nudos reales la presión será incógnita y el caudal dato, en los ficticios pasará a ser justo al contrario. El coeficiente de la válvula se supondrá dato, siendo su valor determinado mediante un calibrado del modelo en el estado de carga valle.
El caudal de fugas de la zona de influencia será, para un estado de carga concreto, función de la diferencia de presiones entre una pareja de nudos representantes de la zona y del coeficiente de la válvula simuladora del conjunto de defectos; la expresión que relaciona estas magnitudes es:

$$
\mathrm{Q}_{\mathrm{f}, \mathrm{i}}=\mathrm{K}_{\mathrm{i}} \cdot \sqrt{\mathrm{p}_{\mathrm{i}}-\mathrm{p}_{\mathrm{sal}}}
$$

donde:

$\mathbf{Q}_{\text {ri }}$ es el caudal de fugas correspondiente a la zona de influencia del nudo i.

$\mathbf{K}_{\mathbf{i}}$ es el coeficiente de la válvula simuladora del comportamiento del conjunto de defectos de la zona i.

$\mathbf{p}_{\mathbf{i}}$ es la presión manométrica del nudo real i.

$\mathbf{p}_{\text {sal }}$ es la presión de salida, que en caso de descarga libre a la atmósfera vale cero.

La expresión (15) es bastante simple, pero simula adecuadamente la realidad, pudiéndose en general mejorar modificando convenientemente el exponente de la diferencia de presiones, el cual varía entre 0.5 y 1.1 .
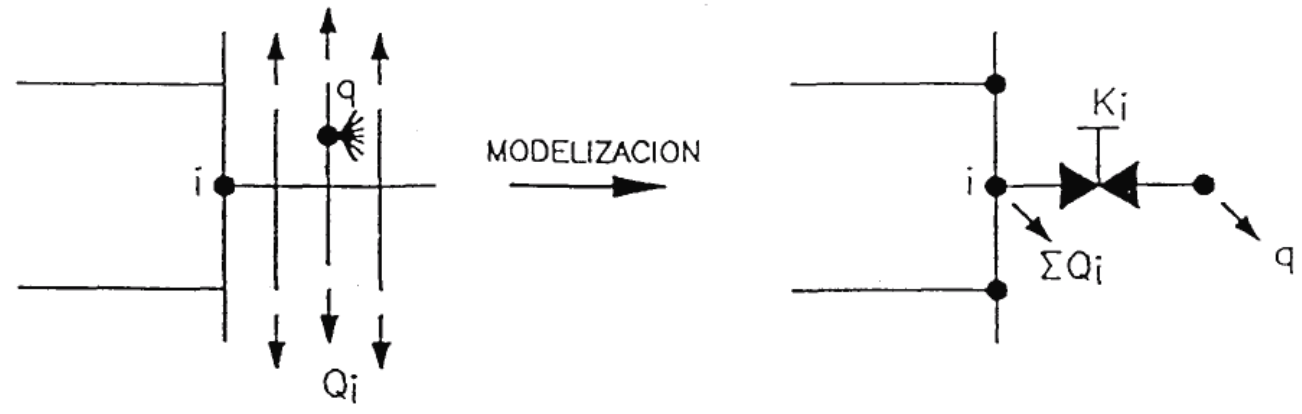

Figura 5. Modelización matemática de fugas en el área de influencia del nudo i.
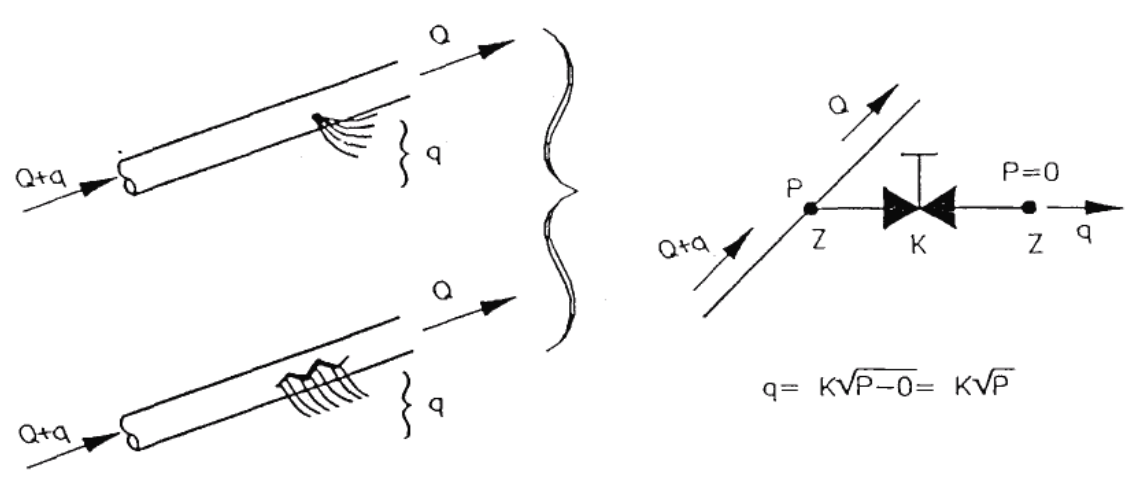

Figura 6. Incorporación de los defectos mediante la inserción de una válvula. 


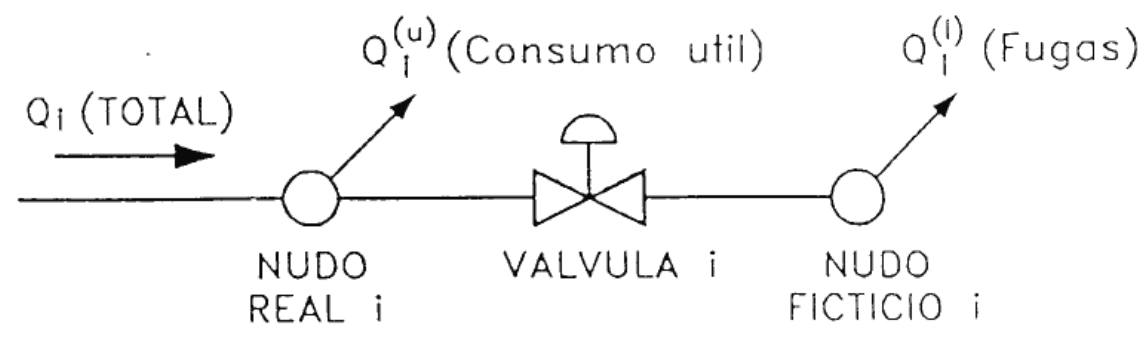

Figura 7. Desdoblamiento de cada nudo del modelo e incorporación de una válvula simuladora equivalente al conjunto de defectos.

Una vez explicado el procedimiento de incorporar los defectos existentes en una determinada red de distribución de agua, procede resolver el problema de su ubicación en la misma. Para ello habrá que asignar unos caudales de fuga a los nudos ficticios para un estado de presiones dado y deducir procediendo a la inversa los valores de $\mathrm{K}_{\mathrm{i}}$ correspondientes según:

$$
\mathrm{K}_{\mathrm{i}}=\frac{\mathrm{Q}_{\mathrm{f}, \mathrm{i}}}{\sqrt{\mathrm{p}_{\mathrm{i}}-\mathrm{p}_{\mathrm{s}}}}
$$

Dado que el número de defectos es variable, el problema de fondo radica en distribuir en el espacio y en el tiempo un conjunto de defectos entre los nudos de la red, según unos criterios de reparto estadístico y registros históricos. Se han propuesto varias formulaciones para estimar el número esperado de roturas en función de los parámetros seleccionados. Como ejemplo citaremos una de estas expresiones:

$$
\mathrm{N}=\frac{\mathrm{K} \cdot \mathrm{L} \cdot \mathrm{e}^{\mathrm{a} \cdot \mathrm{t}}}{\mathrm{D}^{\mathrm{b}}}
$$

donde:

$\mathbf{N}$ es el número esperado de roturas en el intervalo $[0, t]$. $\mathbf{K}$ es un coeficiente característico del material, tipo y calidad de la instalación.

L es la longitud.

D es el diámetro, siendo su exponente, $b$, de valor - 1 para diámetros pequeños hasta $100 \mathrm{~mm}$, y de valor 1 para diámetros mayores.

a es un parámetro que ajusta la evolución temporal.

\section{Simulación del comportamiento de una red con fugas}

Incorporados los defectos al modelo de la red con el procedimiento expuesto, el caudal de fugas podrá ser evaluado por el algoritmo de cálculo para cada estado de carga solicitado, pudiendo estimar el impacto de la regulación y el control de la red en el volumen de fugas, así como la influencia de otros muchos factores tales como:

\author{
- $\quad$ El régimen de presiones. \\ - $\quad$ La demanda de caudal. \\ - $\quad$ La distribución de caudales entre las líneas de la \\ red. \\ - $\quad$ El grado de mallado. \\ - $\quad$ El aumento de la rugosidad y la disminución de \\ diámetros con el tiempo.
}

Pueden hacerse entonces diferentes tipos de simulaciones, modificando presiones de inyección, posiciones de válvulas de regulación, consumos útiles etc. y el modelo analizará las situaciones resultantes recalculan-do de nuevo :

- $\quad$ Presiones en los nudos.

- $\quad$ Caudales circulantes en las líneas.

- Los nuevos caudales de fuga en esas situaciones.

También es conveniente determinar las presiones máximas y mínimas resultantes de los análisis de posibles fallos y accidentes en el sistema, por supuesto en régimen transitorio, para seleccionar los dispositivos de protección más convenientes y evitar situaciones indeseadas provocadas como consecuencia de dichos sucesos. La simplificación frecuente de analizar éstos u otros modos de funcionamiento $\mathrm{y}$ de respuesta del sistema mediante el simple cálculo en régimen permanente, no es adecuada, y como puede fácilmente comprobarse impide detectar depresiones (incluso cavitación) y sobrepresiones que pueden hacer peligrar la integridad física del sistema, ocasionando más fallos posteriormente al suceso inicial, lo que se denomina "cascada de fallos", y que se presenta con bastante frecuencia.

Metodología de localización y cuantificación de caudales de fuga con la ayuda de un modelo matemático apropiado

Aunque en el epígrafe anterior se ha propuesto la asignación previa de unos caudales de fuga en los nudos ficticios para determinar los valores de $\mathrm{K}_{\mathrm{i}}$ en realidad la detección y localización de fugas en sistemas de distribución de agua puede ser acometida 
mediante la resolución del problema denominado inverso, utilizando como datos adicionales medidas de presión y caudal. El problema sobredeterminado (más medidas que las estrictamente necesarias para resolver y localizar las fugas) es el que mejores resultados proporciona y el más fiable. Asimismo, conviene resaltar que la disminución de presiones ocasionada por la presencia de fugas medianas no es importante y es difícil detectarlas en base a esa disminución, siendo necesarias medidas de presión y caudal simultáneamente.

Este método no sustituye a otros sino que sirve como una guía orientativa y complemento de los siempre necesarios métodos tradicionales. La eficacia de este método, como la de otros, en general, dependerá fundamentalmente de:

a) La configuración del sistema (topología).

b) La precisión de las medidas (presión y caudal).

c) La incertidumbre en el conocimiento de las características del sistema.

d) La precisión en la evaluación de las demandas útiles.

La resolución del problema inverso es conveniente realizarla mediante la consideración de régimen transitorio, en base a los mismos métodos que se emplean para la resolución del problema directo, resultando fundamental en el caso de ocurrencia súbita de roturas para su detección y localización, lo que resultaría casi imposible de detectar suponiendo simplemente una simple secuencia de regímenes permanentes en el tiempo.

Los resultados del método inverso consisten en:

- $\quad$ Presiones en los nudos restantes de la red.

- Caudales circulantes en todas las líneas.

- Relación de los nudos que presentan fugas (aquellos con $\mathrm{k}_{\mathrm{j}}>0$ ).

- $\quad$ Caudales de fuga en dichos nudos.

\section{Explotación de los modelos matemáticos de simulación de fugas}

Confeccionado y calibrado el modelo matemático éste puede ahora explotarse para conseguir los objetivos básicos que se pretenden en un abastecimiento:

a) Garantizar una calidad de servicio a los abonados, consistente en el suministro del caudal demandado con un nivel de presiones comprendido en un rango adecuado, con un grado de disponibilidad aceptable.

b) Disminuir el coste total que supone dicho abastecimiento a los abonados cumpliendo el objetivo múltiple expuesto en el apartado a).

Una de las estrategias fundamentales para cumplir dichos objetivos y alcanzar el estado óptimo es, sin duda, reduciendo el volumen no controlado. Esto supone primero conocer bien la magnitud de dicho volumen no controlado, su composición, las causas que lo han producido y su comportamiento en función del estado del sistema.

Fundamentalmente existen dos técnicas para reducir ese volumen de pérdidas:

a)

Aquellas que pretenden restaurar y mantener el sistema, para evitar que el volumen perdido sea importante y aumente de valor, al hacerlo el número de fugas y roturas en el sistema.

b) Otras asumen los defectos ya existentes como latentes, al ser difícilmente localizables y de reparación poco rentable, e intentan reducir las pérdidas volumétricas mediante un control $\mathrm{y}$ regulación óptimo del sistema.

Ambas técnicas son recomendables para todo sistema de abastecimiento, siendo en realidad complementarias, y además emplean herramientas y recursos comunes:

- $\quad$ Explotación del modelo matemático de la red

- $\quad$ Reducción de presiones excesivas.

El uso de un sólo tipo de técnicas, no es suficiente para garantizar un estado óptimo en la red; el mantenimiento preventivo y correctivo debe englobar gran parte de las mismas, y además una operación adecuada en la red, no sólo permite reducir pérdidas sino prevenir accidentes.

En el caso particular de la puesta en práctica de restricciones de agua, el planteamiento anterior adquiere mucho interés, puesto que la interrupción completa de suministro presenta los siguientes inconvenientes:

- Introducción de sustancias nocivas procedentes del medio circundante que contaminarían después el agua potable. Este fenómeno ha ocurrido en algunas poblaciones, en las que concurrían estado deficiente de la red de saneamiento y cortes de suministro en el abastecimiento.

- Introducción de aire dentro de los elementos constituyentes de la red lo que puede resultar muy peligroso al restaurarse el suministro.

- $\quad$ Posible rotura de conducciones al producirse la depresión.

Por tanto, debe evitarse la interrupción completa del suministro, manteniendo al menos una presión mínima $\mathrm{Pi}<\mathrm{P}_{0}$, lo que se traduce en un caudal perdido durante el periodo de restricción. No obstante, el agua inyectada podría ser en ese intervalo de una calidad menor, incluso sólo sanitariamente permisible y no necesariamente potable. 
La red hidráulica de transporte y distribución de agua se puede entender también, o interpretar, como un sistema de transporte de energía. Este es un punto de vista muy interesante y que además complementa perfectamente la concepción tradicional de las redes como sistemas de transporte de agua. El interés primordial de este planteamiento consiste en encontrar las prestaciones máximas de la red, que se traducen en la transferencia máxima de potencia útil, con la restricción de un rendimiento volumétrico aceptable. En muchas redes, debido a la presencia de fugas, y a la excesiva pérdida de carga en el transporte, su rendimiento energético es muy bajo y la potencia útil también, obligando a los usuarios a realizar fuertes inversiones en grupos de sobreelevación. Estos grupos ocasionan gastos energéticos y de mantenimiento importantes y podrían reducirse total o parcialmente si se mejorará el sistema y se reducieran las fugas.

\section{CONCLUSIONES FINALES}

Los planteamientos expuestos de los diferentes problemas tratados, así como los métodos para analizarlos y resolverlos adecuadamente desde la perspectiva de la optimización de la distribución, ofrece bastantes novedades respecto a los planteamientos tradicionales, que presentan a nuestro entender deficiencias serias debido a una excesiva simplificación. No obstante, y como primera aproximación pueden considerarse algunos criterios, que en base al nivel de fugas y al coste del metro cúbico de agua perdido recomiendan usar uno u otro método determinado, como el que muestra la figura siguiente:

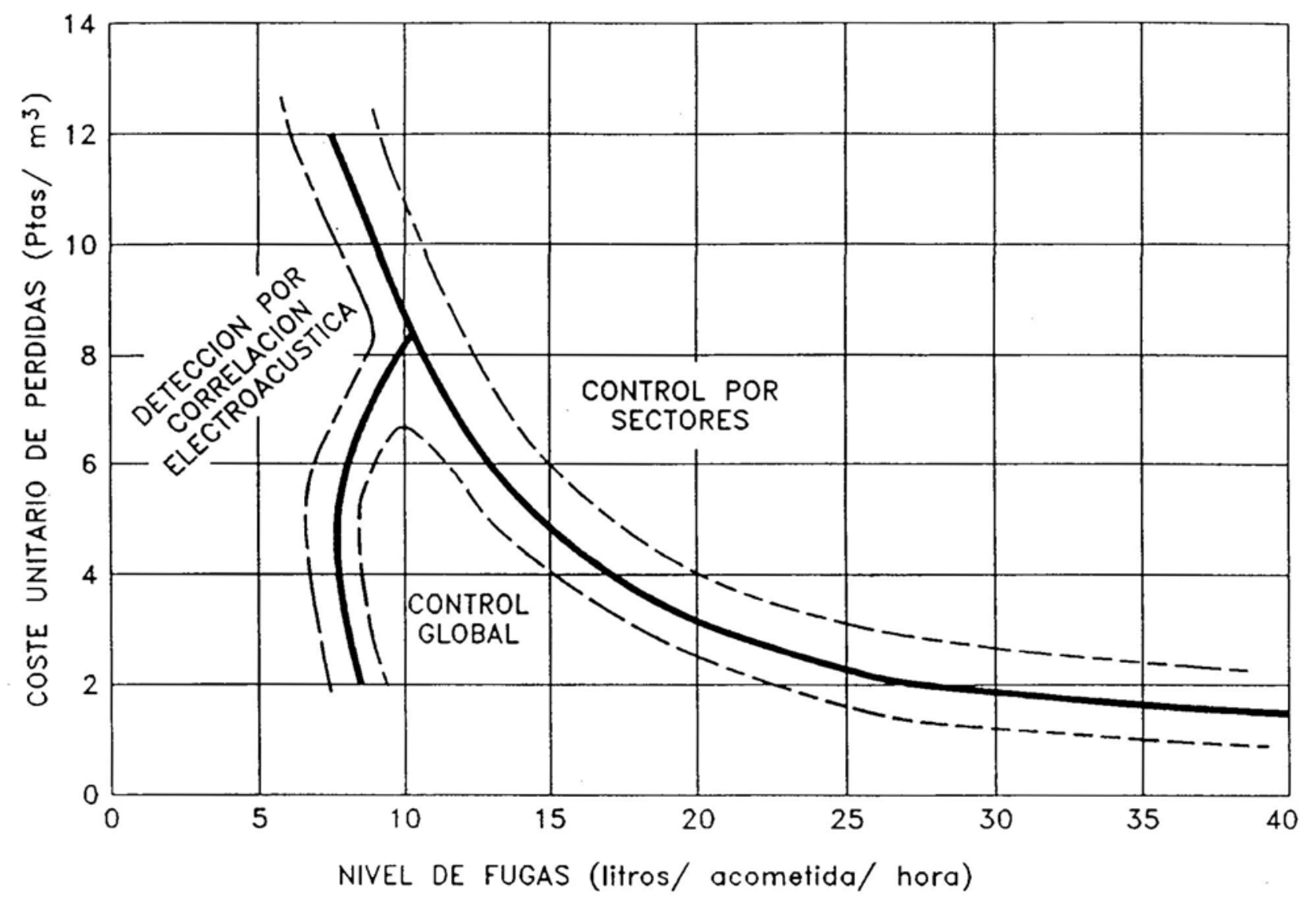

Figura 8. Selección del melado adecuado de delección de fugas (Valerio, 1988)

Al margen de criterios tan simplistas resulta fundamental efectuar un buen diagnóstico de las instalaciones que constituyen un abastecimiento, etapa previa imprescindible para su mejora, tanto en términos de calidad de servicio, como de eficacia y eficiencia.
Tanto en la etapa de diagnóstico, como en la de selección de mejoras y finalmente en la toma de 
decisiones, resulta de inestimable ayuda esa poderosa herramienta que es el modelo matemático, la cual sumada a la experiencia y criterio de los responsables de la gestión y explotación, es cada vez más necesaria. El modelo matemático de dichos sistemas de abastecimiento constituye un verdadero reflejo de lo que es, puede ser y debiera ser el sistema "óptimo" desde los diferentes criterios empleados para su concepción, regulación y mantenimiento.

En particular la importancia del análisis de riesgos, bajo la incertidumbre que preside siempre ese análisis y para los diferentes escenarios que pueden considerarse, es solamente abordable, con rigor, desde la modelización matemática.

En nuestro país, son todavía pocos los abastecimientos que disponen de una actualizada información sobre los aspectos anteriores, y éste es sin duda un grave problema y un importante obstáculo para mejorar sus respectivos servicios y alcanzar los múltiples objetivos que deben plantearse, tanto de índole técnica como económica.

En resumen, la mejora de los ratios indicadores de la eficiencia de todo sistema de abastecimiento requiere entre otros objetivos disminuir el volumen de agua no registrado hasta que dichos parámetros sean aceptables, lo que exige:

1. Aumentar el volumen registrado instalando contadores adecuados y estableciendo un programa óptimo de renovación de los mismos.

2. Disminuir el volumen no aprovechado correctamente o simplemente perdido, mediante la implantación de un programa de mejora integral que coordine las estrategias óptimas seleccionadas para tal fin.

3. Reducir el riesgo de ocurrencia de fallos, roturas y pérdidas futuras de agua, planificando adecuadamente las inversiones en seguridad, calidad de elementos, periodos óptimos de renovación y recurriendo al mantenimiento preventivo.

Muchas veces las nefastas consecuencias derivadas de la escasez de un agua de buena calidad, elemento vital para el desarrollo de nuestra sociedad, se acrecientan más con la "sequía tecnológica" imperante que por la sequía hidrológica en sí. Esta última, al ser transitoria por naturaleza, terminará y en cambio la primera es en muchos casos permanente.

Finalmente terminaremos recordando que no siempre debe privar el criterio económico en las actuaciones de mejora, debiendo tenerse en cuenta otros como garantizar una fiabilidad y eficiencia mínimas en los abastecimientos de agua actuales, aunque aumente el coste global.

\section{REFERENCIAS}

A.E.A.S. (1990) "El Suministro de Agua Potable en España -1987" Documento técnico publicado por la Asociación Española de Abastecimiento y Saneamiento.

A.E.A.S. (1992) "El Suministro de Agua Potable en España -1990" Documento técnico publicado por la Asociación Española de Abastecimiento y Saneamiento.

Cabrera E., Andrés M., Planells F. (1994) "Optimal Management of Pipe Network Maintenance by Cost Analysis of Water Losses". Journal AWWA, aceptado para su publicación, Julio 1994

Cabrera, E. (1989) "Régimen de Explotación Óptimo en un Abastecimiento de Agua" El Agua en la Comunidad Valenciana. Generalitat Valenciana.

Cascetta F., Vigo P. (1992) "Location and assessment of water leakage" Measurement + Control, Volume 25, November 1992.

Cesario, Al (1991) "Network Analysis for Planning, Engineering, Operations and Management Perspectives" Journal AWWA, pp 38-42, February 1991.

Coulbeck, B. (1988) "Computer Applications in Water Supply" Volume 1 - Systems Analysis and Simulations.

Germanopoulos G. (1989) "Leakage reduction by excess pressure minimization in a water supply network" Proc. Instn. Civil Engrs. Part. 2. Paper 9404.

Goulter I.C., Kazemi A. (1989). "Analysis of water distribution pipe failure types in Winnipeg, Canadá" Journal of Transportation Engineering. Vol. 115. $\mathrm{n}^{\circ} 2$.

Jowitt P. (1990) "Optimal valve control in water distribution networks" Journal of Water Resources Planning and Management. Vol. 116, No. 4.

Khadam M., Shammas N. (1991) "Water Losses from Municipal Utilities and their impacts" 1WRA, Water International, 16, No 4, 254-261.

Koelle, E. (1989) "Modelización y control de redes de distribución de Agua Potable" El Agua en la Comunidad Valenciana. Generalitat Valenciana y U.P.

Valencia. 
Liggett, J. (1992) "Network monotoring and the algorithmic location of leaks under steady conditions" Los abastecimientos de agua urbanos. Estado actual y tendencias futuras. UIMP. Valencia.

Lord W., Chase J.(1983)."Choosing the optimal water conservation policy" Journal AWWA. Management and Operations. pp 324-329.July 1983.

Male J.W. Noss R.R. (1985). "Identifying and reducing losses in water distribution systems" Noyes Publications. New Jersey.

Martínez F. (1989) "Modelización y diseño de redes de distribución de agua" El agua en la Comunidad Valenciana. Generalitat Valenciana.
Shamir U., Howard C.D.D. (1979) "An Analytical Approach to Scheduling Pipe Replacement" Journal AWWA, 71:5:248.

Valerio A., Giles H. (1988) "A Simple Economic Model For The Selection of The Optimum Method of Leakage Control" Water International, 13 92-97.

Vela A. (1993) Contribución a la diagnosis y optimización del mantenimiento integral de sistemas de distribución de agua. Tesis Doctoral. Universidad Politécnica de Valencia.

Walski T.M. (1984) Analysis of Water Distribution Systems. Van Nostrand Reinhold. New York.

Walski T.M. (1987) "Replacement Rules for Water Mains " Journal AWWA. Management and Operations. pp 33-37. November 1987. 